\title{
INDUCED MAPS FOR POSTNIKOV SYSTEMS $\left({ }^{(1,2)}\right.$
}

\author{
BY \\ DONALD W. KAHN
}

In the fundamental work of Postnikov $[12]\left({ }^{3}\right)$ and Zilber (see the reference in [17]), one decomposes a space into a sequence or tower of fibre spaces, each of which has only a finite number of nonvanishing homotopy groups. A similar construction in the category of semisimplicial complexes was the basic technique. The procedure involved choices and was not functorial. In the semisimplicial case, J. C. Moore [10] described the construction of a natural Postnikov system for a Kan complex. In practice, his definition involves the following two difficulties: (1) To reconstruct the geometric case, one must take the singular complex of a space, perform the construction, and then apply the geometric realization functor [7]. The resulting objects are not fibre bundles. It is not clear how to describe them by means of invariants. (2) To obtain useful invariants to describe the fibre spaces in the construction, one must replace the fibre spaces by twisted Cartesian products. This process involves choices, and one no longer has a natural object.

In this note, I consider Postnikov systems from a geometric point of view (see [5]). The principal result is, roughly speaking, the following:given a map of spaces $f: X \rightarrow Y$, there is an induced map of Postnikov systems, which sends each term in a Postnikov decomposition for $X$ into the corresponding term in a Postnikov decomposition for $Y$. These individual maps are all compatible. Furthermore, the invariants ( $k$-invariants) for $X$ and for $Y$ are related via the map $f$. I believe that this construction will serve as an adequate substitute for a functorial Postnikov construction, which in the geometric case seems unlikely for technical reasons.

There are three sections. The first is preliminary, while the second is concerned with the actual construction. In the third section, I give several applications to $H$-spaces, including a characterization of $H$-spaces with finitely many nonvanishing homotopy groups. In a subsequent note, I plan to study the group of homotopy equivalences of a space.

Received by the editors June 18, 1962.

(1) Work supported by contract NONR-266 (57).

(2) Added in proof. After this paper was written, I learned that many of these results were known to Hilton, Ganea and others. However, I believe that the main results of this paper are not available elsewhere in print.

(3) Reference to Bibliography at end of paper. 
I would like to thank A. Dold, both for many stimulating conversations, and for giving me Proposition 3.1.

1. Preliminaries. In this paper we assume that all spaces have base points. A map $f: X \rightarrow Y$ shall be a continuous map which sends the base point in $X$ into the base point in $Y$. Therefore, homotopies will leave the base point fixed. If $X$ is a space, $P X$ will denote the space of paths beginning at the base point in $X$, while $\Omega X$ will denote the subspace of $P X$ consisting of those paths which also end at the base point. $P X$ is given the compact open topology. If $f: X \rightarrow Y$ is a map, then we have induced maps $P f: P X \rightarrow P Y$ and $\Omega f: \Omega X \rightarrow \Omega Y$.

Definition 1.1. Let $E$ and $B$ be spaces and $p: E \rightarrow B$ be a map. We say that $(E, B ; p)$ satisfies the absolute covering homotopy property (ACHP), and that $E$ is a fibre space over $B$, if for any space $X$ and map $G: X \times I \rightarrow B$, and a map $\bar{H}: X \times\{0\} \rightarrow E, p \cdot \bar{H}=G \mid X \times\{0\}$, there is a map $H: X \times I \rightarrow E$ which extends $\bar{H}$ and satisfies $p \cdot H=G$. (Note that we require $G$ and $H$ to map the set (base point $\times I$ ) into a point. The base point of $X \times I$ will be taken to be (base point $\times\{0\})$.)

If we define the map $p: p X \rightarrow X$ to send each path onto its endpoint, then $(P X, X ; p)$ is a fibre space (see p. 479 of $[14])$.

Now suppose $(E, B ; p)$ is a fibre space and $f: X \rightarrow B$ is a map. Define $f^{-1} E=\{(x, e) \in X \times E \mid f(x)=p(e)\}$, and $\bar{p}: f^{-1} E \rightarrow X$ by $\bar{p}(x, e)=x$. Then it is easy to see that $\left(f^{-1} E, X ; \bar{p}\right)$ is a fibre space (see, for example, [14]). It is called the induced fibre space over $X$. If we define $\bar{f}: f^{-1} E \rightarrow E$ by $\bar{f}(x, e)=e$, then it is clear that the diagram

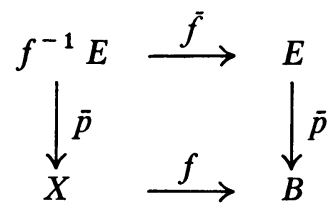

is commutative. We say that $(\tilde{f}, f)$ is a map of fibre spaces. If $(E, B ; p)$ is a fibre space, and $b \in B$ is the base point, then we refer to $p^{-1}(b)$ as the fibre. When required for clarity, we write $(E, F, B ; p)$ instead of $(E, B ; p)$.

Now, let $f: X \rightarrow Y$ be a map, and let $Y^{I}$ be the space of maps $I \rightarrow Y$ with the compact-open topology. We take as the base point the constant map from $I$ to the base point of $Y$. Define $X_{f}=\left\{(x, p) \in X \times Y^{I} \mid f(x)=p(1)\right\}$, and define $p_{f}: X_{f} \rightarrow Y$ by $p_{f}(x, p)=p(0)$. It is easy to verify that $\left(X_{f}, Y ; p_{f}\right)$ is a fibre space, that $X_{f}$ has the homotopy type of $X$, and that

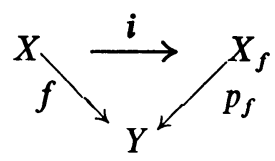

is a commutative diagram, where $i$ is the obvious inclusion. 
Definition 1.2. If $f: X \rightarrow Y$ is a map, we say that $\left(X_{f}, Y ; p_{f}\right)$ is the fibre space obtained from $f$, or that $p_{f}$ is the replacement of $f$ by a fibre map.

Notice that in this definition we give an explicit fibre space, and that the base space is $Y$.

It is easy to verify that a finite composition of fibre maps is a fibre map, and that if one replaces a sequence or composition of maps by a fibre map, the result is homotopic, in a natural way, to the composition of fibre maps obtained by replacing each map by a fibre map.

For the rest of this paper, we shall consider spaces having the homotopy type of a countable, 1-connected, CW-complex (with base point). Denote the category of all such spaces, with (base point preserving) maps, by $\mathscr{C}$. Milnor [9] has given several descriptions of the spaces in this category; his results show that the constructions which will be performed in the present paper do not lead us outside of the category $\mathscr{C}$. This point is essential only in considering the classification of fibre spaces over some given space. An alternative approach would be to use the theory of numerable bundles, which is due to A. Dold (not yet published).

LeMMA 1.1. If $X$ and $Y$ belong to $\mathscr{C}$, and if $f: X \rightarrow Y$ induces isomorphisms of all homotopy groups, i.e., $f_{\#}: \pi_{i}(X) \cong \pi_{i}(Y)$ for all $i \geqq 0$, then $f$ is a homotopy equivalence.

Proof. See [18]. This is an immediate consequence of J. H. C. Whitehead's theorem, which is the same except that $X$ and $Y$ are taken to be complexes.

LEMMA 1.2. Let $(E, B ; p)$ be a fibre space, and let $X$ be a space of the homotopy type of $B$. Let $f: X \rightarrow B$ be a homotopy equivalence, and let $\left(f^{-1} E, X ; \bar{p}\right)$ be the induced fibre space. Then the map $\tilde{f}^{-1} f^{-1} E \rightarrow E$ is a homotopy equivalence.

Proof. By considering the induced maps, on homotopy, of $f$ and $\bar{f} \mid \bar{p}^{-1}\left(x_{0}\right), x_{0}$ being the base point, we see that $\vec{f}$ induces isomorphisms in homotopy groups in all dimensions. The conclusion follows from the previous lemma.

If $\pi$ is an abelian group, let $H^{n}(X, \pi)$ denote the $n$th singular cohomology group of $X$ with coefficients in $\pi$.

We will make frequent use of the equivalence between classes in $H^{n}(X, \pi)$ and homotopy classes of maps $X \rightarrow K(\pi, n)$. (See for example, [15].) Let $g: Y \rightarrow X$ be a map. Let $u \in H^{n}(X, \pi)$ be a class and $\bar{u}: X \rightarrow K(\pi, n)$ be the corresponding map (or class of maps). The following lemma is immediate.

LEMMA 1.3. $\overline{g^{*}(u)}=\bar{u} \circ g$.

Now let $\phi: \pi \rightarrow \pi^{\prime}$ be a homomorphism. Denote by $\phi_{c}$ the coefficient homomorphism $H^{n}(X ; \pi) \rightarrow H^{n}\left(X ; \pi^{\prime}\right)$ and by $\tilde{\phi}$ a map $K(\pi, n) \rightarrow K\left(\pi^{\prime}, n\right)$ which induces $\phi$ on the $n$th homotopy group.

LEMMA 1.4. If $u \in H^{n}(X, \pi)$, then $\left\{\overline{\phi_{c}(u)}\right\}=\{\tilde{\phi} \cdot \bar{u}\}$. 
Proof. Consider the maps

$$
X \stackrel{\bar{u}}{\rightarrow} K(\pi, n) \stackrel{\tilde{\phi}}{\rightarrow} K\left(\pi^{\prime}, n\right) .
$$

As $H^{n}\left(\pi^{\prime}, n ; \pi^{\prime}\right)=\operatorname{Hom}\left(\pi^{\prime}, \pi^{\prime}\right)$, we let $i^{\prime} \in H^{n}\left(\pi^{\prime}, u ; \pi^{\prime}\right)$ be the identity isomorphism. Clearly $\tilde{\phi}^{*}\left(i^{\prime}\right)$ is the homomorphism $\phi$. Now consider the commutative diagram

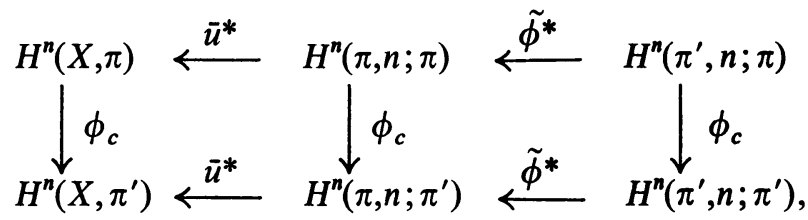

$\phi_{c}(i)=\tilde{\phi^{*}}\left(i^{\prime}\right)$ because both these classes identify with the homomorphism $\phi$. Now $\phi_{c} \bar{u}^{*}(i)=\bar{u}^{*} \phi_{c}(i)$ by the left square of the diagram. Therefore, $\phi_{c} \bar{u}^{*}(i)=\bar{u}^{*} \tilde{\phi}^{*}\left(i^{\prime}\right)$. Hence, the class of maps associated with $\phi_{c} \bar{u}^{*}(i)=\phi_{c}(u)$ is $\{\tilde{\phi} \cdot \bar{u}\}$.

We now define a Postnikov system.

Definition 1.3. Let $X \in \mathscr{C}$. A Postnikov system for $X$ is a family of spaces $X_{i}, i \geqq 0$ along with maps

$$
\begin{aligned}
& p_{i}: X \rightarrow X_{i} \\
& \pi_{i, j}: X_{i} \rightarrow X_{j}, \quad i>j \quad\left(\text { if } i>j>k, \pi_{i, k}=\pi_{j, k} \pi_{i, j}\right)
\end{aligned}
$$

such that

1. If $X$ is $(n-1)$-connected, each $X_{i}, 0 \leqq i \leqq n-1$,consists of a single point, the base point.

2. $p_{i}: X \rightarrow X_{i}$ induces isomorphisms in homotopy in dimensions $\leqq i$.

3. $\pi_{i+1, i}: X_{i+1} \rightarrow X_{i}$ is a fibre map, with fibre $K\left(\pi_{i+1}(x), i+1\right)$.

4. $\pi_{i+1, i} \cdot p_{i+1} \simeq p_{i}$.

For the construction of such a system in the category of semi-simplicial complexes, see [10]. For the method of construction in the geometric case, see [5].

For convenience of notation, we assume that if $\pi=\{e\}$, the trivial group, then $K(\pi, n)$ consists of a single point.

Definition 1.4. Let $X, X^{\prime} \in \mathscr{C}$. Let $X$ and $X^{\prime}$ have Postnikov systems $\left\{X_{i}, p_{i}, \pi_{i, j}\right\}$ and $\left\{X_{i}^{\prime}, p_{i}^{\prime}, \pi_{i, j}^{\prime}\right\}$. Let $f: X \rightarrow X^{\prime}$ be a map. Then, by an induced map on the Postnikov systems for $X$ and $X^{\prime}$, we mean a family of maps $f_{i}: X_{i} \rightarrow X^{\prime}{ }_{i}$, such that for each $i, f_{i} \cdot \pi_{i+1, i}=\pi_{i+1, i}^{\prime} \cdot f_{i+1}$ and $p_{i}^{\prime} \cdot f \simeq f_{i} \cdot p_{i}$.

\section{Construction of the induced maps.}

Definition 2.1. (1) If $X$ is a space, we say that $X^{\prime}$ is equivalent to $X$, if $X^{\prime}$ has the homotopy type of $X$.

(2) If $f: X \rightarrow Y$ is a map, and if $X^{\prime}$ and $Y^{\prime}$ are equivalent to $X$ and $Y$ (resp.), then a map $f^{\prime}: X^{\prime} \rightarrow Y^{\prime}$ is equivalent to $f$, if there is a homotopy commutative diagram 


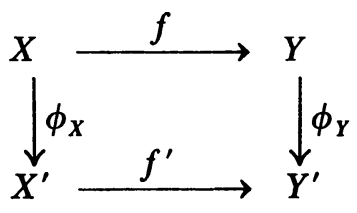

where $\phi_{X}$ and $\phi_{Y}$ are homotopy equivalences.

Proposition 2.1. Given a homotopy commutative diagram

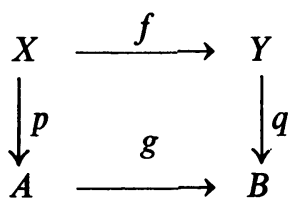

there are spaces $X^{\prime}, Y^{\prime}, A, B^{\prime}$ which are equivalent to $X, Y, A, B$, and maps $f^{\prime}, g^{\prime}, p^{\prime}, q^{\prime}$ which are equivalent to $f, g, p, q$ such that (1)

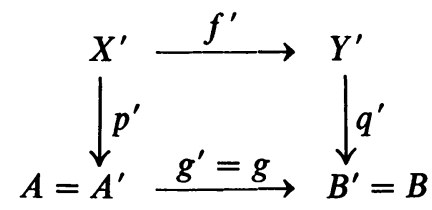

is (strictly) commutative and (2) $p^{\prime}$ and $q^{\prime}$ are fibre maps. In other words, $\left(f^{\prime}, g^{\prime}\right)$ is a map of fibre spaces.

Proof. Convert the maps $p$ and $q$ to fibre maps, $p^{\prime}: X^{\prime} \rightarrow A$, and $q^{\prime}: Y^{\prime} \rightarrow B$. We then easily obtain a homotopy commutative diagram

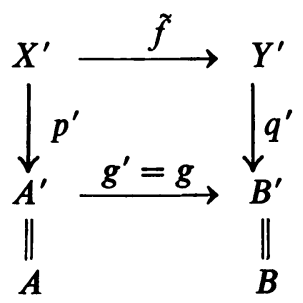

The map $q^{\prime} \cdot f: X^{\prime} \rightarrow B^{\prime}$ is covered by the map $f: X^{\prime} \rightarrow Y^{\prime}$. But $q^{\prime} \cdot \tilde{f} \simeq g^{\prime} \cdot p^{\prime}$. We cover the homotopy, getting a map $f^{\prime}: X^{\prime} \rightarrow Y^{\prime}$ such that $q^{\prime} \cdot f^{\prime}=g^{\prime} \cdot p^{\prime}$.

Now, we suppose that $X$ is $(n-1)$-connected and $Y$ is $(m-1)$-connected, with $n, m>1$. Set $p=\min (n, m)$. Let $p(x): X \rightarrow K\left(\pi_{p}(X), p\right)$ (resp. $\left.p(Y): Y \rightarrow K\left(\pi_{p}(Y), p\right)\right)$ represent the fundamental classes in $X$ (resp. $Y$ ) or the zero class in $X$ (resp. $Y)$, according as $n=p($ resp. $m=p)$ or $n>p($ resp. $m>p)$.

Proposition 2.2. Let $f_{\#}: K\left(\pi_{p}(X), p\right) \rightarrow K\left(\pi_{p}(Y), p\right)$ be induced by $f: X \rightarrow Y$. (See, for example, [15].) Then the diagram 


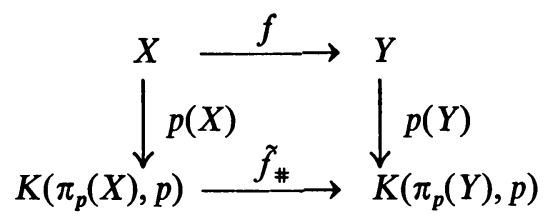

is homotopy commutative.

Proof. CaSe 1. Either $p<n$ or $p<m$. Supposing that $p<n$, then $p(X)$ and $\tilde{f}_{\#}$ are null-homotopic, and as $\pi_{i}(X)=0, i<n$, it follows that $H^{p}\left(X, \pi_{p}(Y)\right)=0$ so that $p(Y) \cdot f$ is also null-homotopic. The case $p<m$ is similar.

CASE 2. $\quad p=n=m . p(X)$ and $p(Y)$ induce the identity isomorphism in homotopy in dimension $p . f$ and $\tilde{f}_{\#}$ induce the same homomorphism in homotopy in dimension $p$. By the Hurewicz theorem, $p(Y) \cdot f$ and $\tilde{f}_{\#} \cdot p(X)$ induce the same homomorphism in homology in dimension $p$. By duality, they induce the same homomorphism in cohomology and also induce the same homomorphism in cohomology with coefficients in $\pi_{p}(Y)$. Therefore, $(p(Y) \cdot f)^{*}(i)=\left(\tilde{f}_{\#} \cdot p(X)\right)^{*}(i)$ or $p(Y) \cdot f \simeq \tilde{f}_{\#} \cdot p(X)$.

Proposition 2.3. With the above notations, there are spaces $X^{\prime}, Y^{\prime}$ which are equivalent to $X, Y$, and maps $p(X)^{\prime}, p(Y)^{\prime}, f^{\prime}$ which are equivalent to $p(X), p(Y), f$ such that

is commutative,

$$
\begin{array}{cll}
X^{\prime} \stackrel{f^{\prime}}{\longrightarrow} & Y^{\prime} \\
\downarrow^{p(X)^{\prime}} & & { }^{p}(Y)^{\prime} \\
K\left(\pi_{p}(X), p\right) \stackrel{f_{\#}}{\longrightarrow} & \stackrel{K}{ } \stackrel{K\left(\pi_{p}(Y), p\right)}{ }
\end{array}
$$

(2) $p(X)^{\prime}$ and $p(Y)^{\prime}$ are fibre maps.

Proof. Apply Proposition 2.1 to Proposition 2.2.

Now, let $F_{X}$ and $F_{Y}$ be the fibres of $p(X)^{\prime}$ and $p(Y)^{\prime}$. Consider the exact sequence

$$
0 \rightarrow \pi_{p}\left(F_{X}\right) \rightarrow \pi_{p}(X) \stackrel{\cong}{\longrightarrow} \pi_{p}(X) \rightarrow \pi_{p-1}\left(F_{X}\right) \rightarrow 0
$$

which is a portion of the homotopy sequence of the fibre space $\left(X^{\prime}, K\left(\pi_{p}(X), p\right)\right)$. We see that $\pi_{p-1}\left(F_{X}\right)=0, \pi_{p}\left(F_{X}\right)=0$. Similarly, we have $\pi_{i}\left(F_{X}\right)=0, i \leqq p$. The dentical argument shows $\pi_{i}\left(F_{Y}\right)=0, i \leqq p$.

Hence, $H^{p+1}\left(F_{X} ; \pi_{p+1}\left(F_{X}\right)\right)$ and $H^{p+1}\left(F_{Y} ; \pi_{p+1}\left(F_{Y}\right)\right)$ contain fundamental classes, which we denote by $i_{X}$ and $i_{Y}$. Denote the transgression, in either fibre space, by $\tau$.

DEFINITION 2.2.

$$
\begin{aligned}
& \tau\left(i_{X}\right)=k_{X}^{p+2}, \text { the first } k \text {-invariant of } X, \\
& \tau\left(i_{Y}\right)=k_{Y}^{p+2} \text {, the first } k \text {-invariant of } Y .
\end{aligned}
$$


Alternative definitions of the $k$-invariant may be given in terms of obstruction theory.

Notice that $\pi_{p+1}\left(F_{X}\right) \approx \pi_{p+1}(X), \pi_{p+1}\left(F_{Y}\right) \approx \pi_{p+1}(Y)$, and that if we identify these groups, $\left(f^{\prime} \mid F_{X}\right)_{\#}: \pi_{p+1}\left(F_{X}\right) \rightarrow \pi_{p+1}\left(F_{Y}\right)$ is identified with $f_{\#}$.

Proposition 2.4. Let $f_{c}^{*}$ denote the coefficient homomorphism induced by $f_{\#}: \pi_{p+1}(X) \rightarrow \pi_{p+1}(Y)$. Then,

$$
f_{c}^{*} k_{X}^{p+2}=\left(\tilde{f}_{\#}^{*} k_{Y}^{p+2}\right.
$$

Proof. Consider the commutative diagram

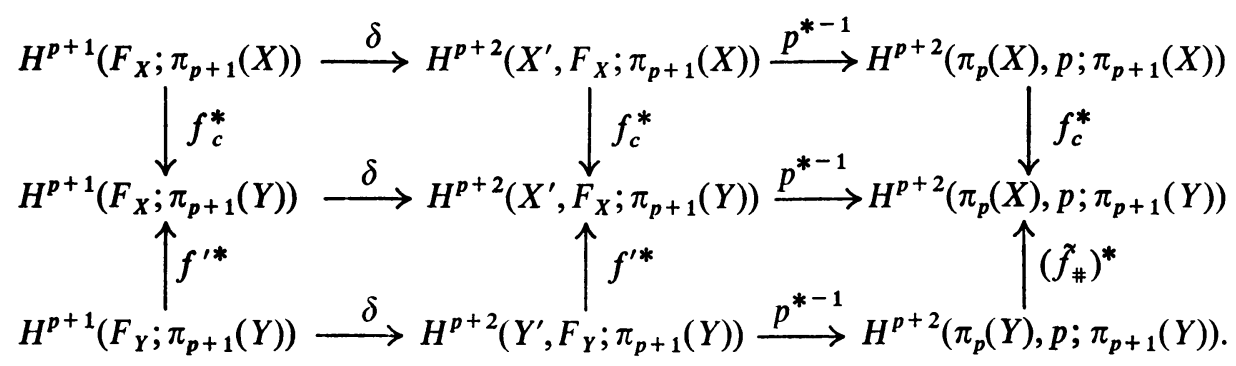

By Lemma $1.4, f_{c}^{*} i_{X} \in H^{p+1}\left(F_{X} ; \pi_{p+1}(X)\right)$ corresponds to the map $\tilde{f}_{\#} \cdot \tilde{\imath}_{X}$ : $F_{X} \rightarrow K\left(\pi_{p+1}(X), p+1\right) \rightarrow K\left(\pi_{p+1}(Y), p+1\right)$. Hence, as an element of $\operatorname{Hom}\left(\pi_{p+1}(Y), \pi_{p+1}(Y)\right)$, it is the map $\pi_{p+1}(X) \stackrel{\text { Id }}{\rightarrow} \pi_{p+1}(X) \stackrel{f_{\#}}{\rightarrow} \pi_{p+1}(Y)$.

By Lemma 1.3, $f^{\prime *} i_{Y}$ corresponds to the homomorphism $\pi_{p+1}(X) \stackrel{f \#}{\rightarrow} \pi_{p+1}(Y)$ $\stackrel{\text { Id }}{\rightarrow} \pi_{p+1}(Y)$, so that $f_{c}^{*} i_{X}=f^{\prime *} i_{Y}$.

Now by the diagram, $f_{c}^{*} k_{X}^{p+2}=f_{c}^{*} \tau\left(i_{X}\right)=f_{c}^{*} p^{*-1} \delta\left(i_{X}\right)=p^{*-1} \delta\left(f_{c}^{*} i_{X}\right)$ $=p^{*-1} \delta\left(f^{\prime *} i_{Y}\right)=\left(\tilde{f}_{\#}\right)^{*} p^{*-1} \delta\left(i_{Y}\right)=\left(\tilde{f}_{\#}\right)^{*} k_{Y}^{p+2}$.

Proposition 2.5. There is a homotopy commutative diagram

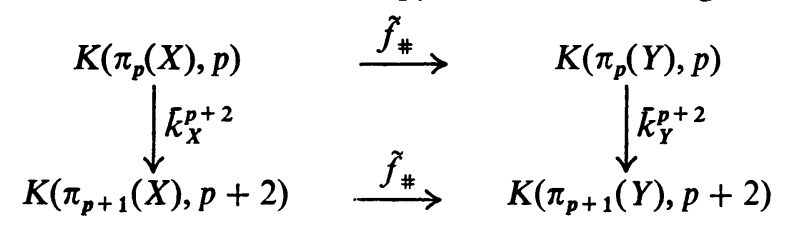

and an equivalent commutative diagram, for which we keep the same notation.

Proof. By Lemmas 1.3 and 1.4, and Proposition 2.4, the compositions $\tilde{f}_{\#} \circ k_{X}^{p+2}$ and $k_{Y}^{p+2} \circ f_{\#}$ are homotopic. To obtain an equivalent commutative diagram, replace the lower $f_{\#}$ by an equivalent fibre map. Then, by the ACHP we may find a map homotopic to $k_{X}^{p+2}$, which makes the diagram commutative.

The main interest in the above proposition is the following:

Proposition 2.6. There are fibre spaces $\left(X_{p+1}, K\left(\pi_{p}(X), p\right) ; \pi_{p+1}^{X}\right)$ and $\left(Y_{p+1}, K\left(\pi_{p}(Y), p\right) ; \pi_{p+1}^{Y}\right)$ with fibres $K\left(\pi_{p+1}(X), p+1\right)$ and $K\left(\pi_{p+1}(Y), p+1\right)$, and a commutative diagram 


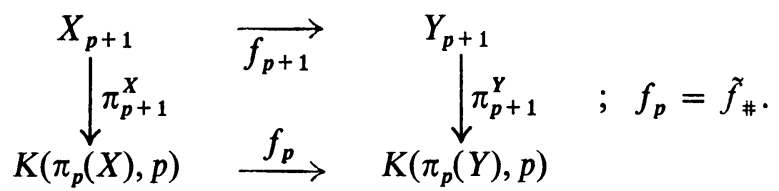

The k-invariants (or characteristic classes) are $k_{X}^{p+2}$ and $k_{Y}^{p+2}$.

Proof. Consider $K\left(\pi_{p+1}(X), p+2\right)$ and $K\left(\pi_{p+1}(Y), p+2\right)$ as classifying spaces for principal bundles with fibres $K\left(\pi_{p+1}(X), p+1\right)$ and $K\left(\pi_{p+1}(Y), p+1\right)$ (resp.) (see [8]). Then the maps $k_{X}^{p+2}$ and $k_{Y}^{p+2}$ induce bundles over $K\left(\pi_{p}(X), p\right)$ and $K\left(\pi_{p}(Y), p\right)$. For our purposes, it is preferable to consider the induced fibre spaces over $K\left(\pi_{p}(X), p\right)$ and $K\left(\pi_{p}(Y), p\right)$, which are obtained by pulling back the corresponding path spaces rather than the universal bundles (see Exposé 1 in [2]). Denoting the path space functor by $P$, we have

$$
\begin{aligned}
& X_{p+1}=\left\{(a, \rho) \in K\left(\pi_{p}(X), p\right) \times P K\left(\pi_{p+1}(X), p+2\right) \mid k_{X}^{p+2}(a)=\rho(1)\right\}, \\
& Y_{p+1}=\left\{(a, \rho) \in K\left(\pi_{p}(Y), p\right) \times P K\left(\pi_{p+1}(Y), p+2\right) \mid k_{Y}^{p+2}(a)=\rho(1)\right\} .
\end{aligned}
$$

Since the diagram in Proposition 2.5 may be assumed to be commutative, we may define $f_{p+1}=\left(\tilde{f}_{\#}, P \tilde{f}_{\#}\right)$. P $\tilde{f}_{\#}$ being the map induced on path spaces, and the diagram is then clearly commutative. The assertion about the $k$-invariants follows immediately from the naturality of the transgression.

REMARKS. Proposition 2.6 represents a map from one 2-stage Postnikov system into another. Furthermore, $\pi_{p+1}^{X}: X_{p+1} \rightarrow K\left(\pi_{p}(X), p\right)$ is a part of a Postnikov system for $X$, because $P(X)^{\prime}$ may be lifted to form a commutative diagram (see [5])

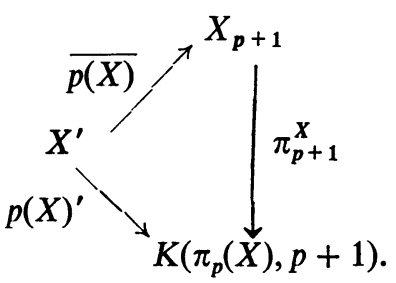

A similar lifting may be performed for $\pi_{p+1}^{Y}: Y_{p+1} \rightarrow K\left(\pi_{p}(Y), p\right)$. In general, we would like the diagram

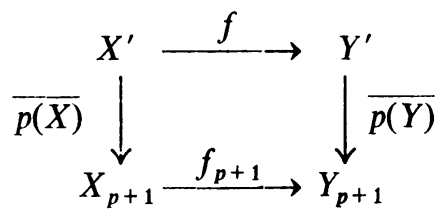

to be commutative, up to homotopy. As the matter stands, this would not be true without some rather strong assumptions (for example, if $\operatorname{Ext}\left(\pi_{p}(X), \pi_{p+1}(Y)\right)=0$ ). The basic point of the following theorem, which is the principal result of this paper, is that we may change the map $f_{p+1}$ in such a way that 


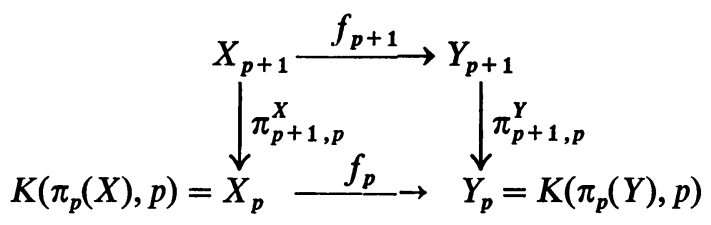

remains commutative, while

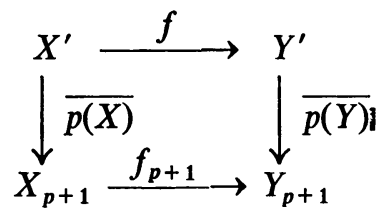

becomes homotopy commutative.

TheOREM 2.1. Let $X, Y \in \mathscr{C}$ and let $f: X \rightarrow Y$ be a map. Then there exis Postnikov systems $\left\{X_{i}, p_{i}^{X}, \pi_{i+1, i}^{X}\right\}$ and $\left\{Y_{i}, p_{i}^{Y}, \pi_{i+1, i}^{Y}\right\}$ for $X$ and $Y$ (resp.) and a diagram

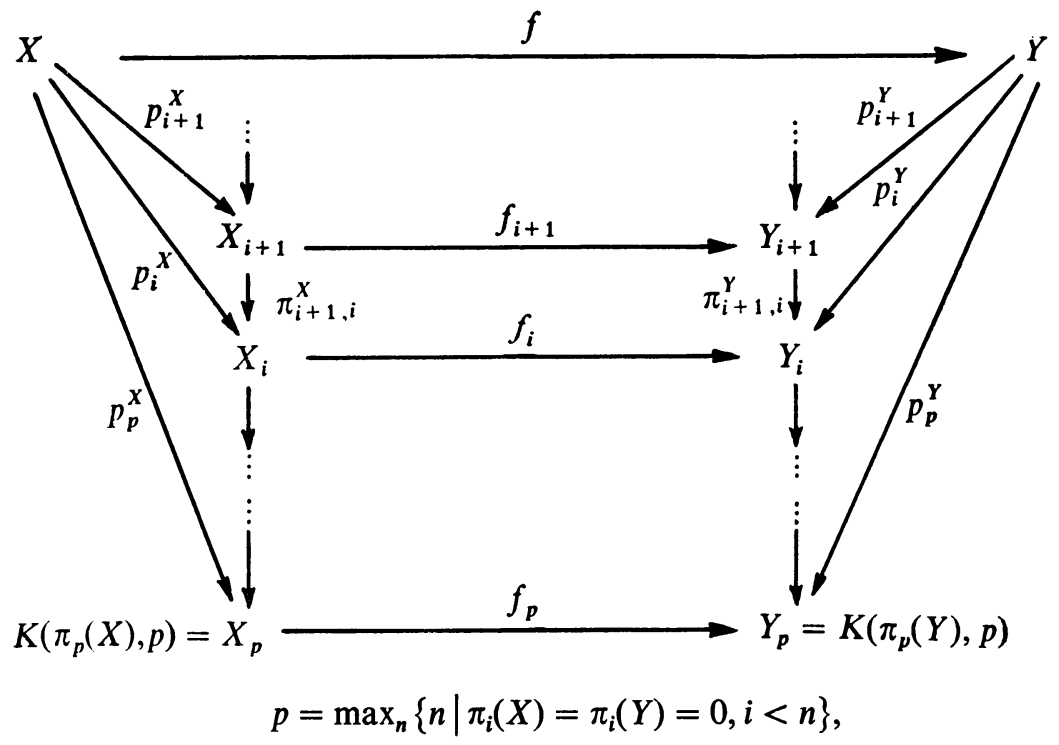

in which all rectangular subdiagrams are (strictly) commutative, and all other subdiagrams are homotopy commutative. For $n \geqq p+2$, denote the $k$-invariants (images of the fundamental classes under transgression) by $k_{X}^{n}$ and $k_{Y}^{n}$. Then,

$$
f_{c}^{*} k_{X}^{n}=f_{n-2}^{*} k_{Y}^{n} \text {. }
$$

Proof. By Propositions 2.3 and 2.4, the theorem is true for one-stage Postnikov decompositions. We shall proceed by induction. Assume that we have constructed Postnikov systems for $X$ and $Y$ consisting of $n$ terms, along with the desired diagram and relation between $k$-invariants. We shall construct spaces $X_{n+1}$ and 
$Y_{n+1}$ and a map $f_{n+1}$ so that the desired conditions are met. The parts of the proof will be marked by letters.

REMARK. At certain places in this proof, we shall need to use the action of the fibre on a fibre space. As all of these fibre spaces are induced from path spaces, they all have the structure of principal fibre spaces (see [11]). The fibres are all topological monoids with homotopy inverses. In order to avoid additional complication, we shall treat these fibre spaces as principal bundles. Actually, it would be possible to carry out the entire discussion using principal bundles, if we were to use a weaker (and more complicated) covering homotopy property than the ACHP. The details of the argument for principal fibre spaces are straightforward, and are left to the reader.

(A) By induction hypothesis,

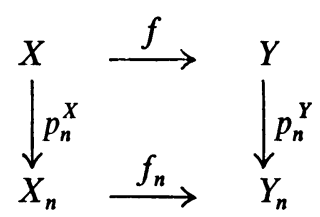

is homotopy commutative. By Proposition 2.1, we may construct an equivalent commutative diagram

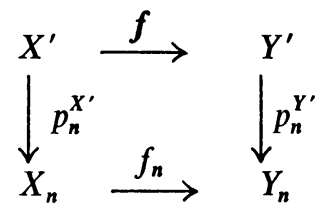

in which the vertical arrows represent fibre maps. Also, by inductive hypothesis, $p_{n}^{X}$ and $p_{n}^{Y}$ induce isomorphisms in homotopy in dimensions $\leqq n$. Denote the fibre of $p_{n}^{X^{\prime}}$ by $F_{n}^{X}$. Then we have the following exact sequence:

$$
\cdots \rightarrow \pi_{n+1}\left(X_{n}\right) \rightarrow \pi_{n}\left(F_{n}^{X}\right) \rightarrow \pi_{n}(X) \underset{\sim}{\approx} \pi_{n}\left(X_{n}\right) \rightarrow \pi_{n-1}\left(F_{n}^{X}\right) \rightarrow \pi_{n-1}(X) \stackrel{\approx}{\rightarrow} \cdots
$$

It follows that $\pi_{i}\left(F_{n}^{X}\right)=0$ for $i \leqq n ; \pi_{n+1}\left(F_{n}^{X}\right) \approx \pi_{n+1}(X)$. Similarly $\pi_{i}\left(F_{n}^{Y}\right)=0$ for $i \leqq n ; \pi_{n+1}\left(F_{n}^{Y}\right) \approx \pi_{n+1}(Y)$. We identify these two groups, and denote the fundamental classes in $H^{n+1}\left(F_{n}^{X} ; \pi_{n+1}(X)\right)$ and $H^{n+1}\left(F_{n}^{Y} ; \pi_{n+1}(Y)\right)$ by $i_{n, X}$ and $i_{n, Y}$. Of course, one defines $k$-invariants as before; in this case $k_{X}^{n+2}=\tau\left(i_{n, X}\right), k_{Y}^{n+2}=\tau\left(i_{n, Y}\right)$.

(B) $f_{c}^{*}\left(k_{X}^{n+2}\right)=f_{n}^{*}\left(k_{Y}^{n+2}\right)$. Exactly as in Proposition 2.4 one sees that $f^{\prime *}\left(i_{n, Y}\right)$ corresponds to the homomorphism $\pi_{n+1}(X) \stackrel{f^{\#}}{\rightarrow} \pi_{n+1}(Y) \stackrel{\text { Id }}{\rightarrow} \pi_{n+1}(Y)$, while $f_{c}^{*}\left(i_{n, X}\right)$ corresponds to the homomorphism $\pi_{n+1}(X) \stackrel{\text { Id }}{\rightarrow} \pi_{n+1}(X) \stackrel{f^{*}}{\rightarrow} \pi_{n+1}(Y)$. Hence, $f^{\prime *} i_{n, Y}=f_{c}^{*} i_{n, X}$. Now, by the naturality of the transgression, one constructs a diagram as was done in Proposition 2.4. The assertion follows immediately. 
(C) As in Proposition 2.5, relation (B) above may be interpreted as a homotopy commutative diagram

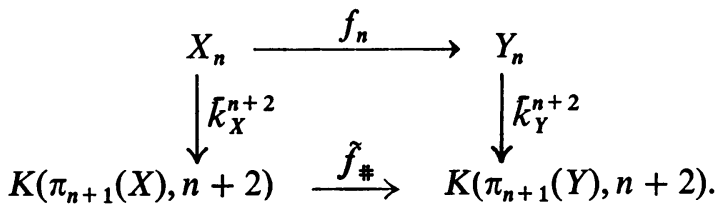

By taking $\tilde{f}_{\#}$ to be a fibre map, we may use the ACHP to change $k_{x}^{n+2}$ into an equivalent map which makes the diagram commutative.

Now, we consider the path spaces over $K\left(\pi_{n+1}(X), n+2\right)$ and $K\left(\pi_{n+1}(Y), n+2\right)$, and define $X_{n+1}$ and $Y_{n+1}$ to be the induced fibre spaces over $X_{n}$ and $Y_{n}$. The respective fibres are $K\left(\pi_{n+1}(X), n+1\right)$ and $K\left(\pi_{n+1}(Y), n+1\right)$. The formula

$$
f_{n+1}(x, a)=\left(f_{n}(x), P \tilde{f}_{\#}(a)\right)
$$

defines a fibre map, so that the diagram

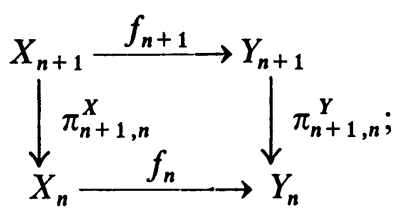

$\pi_{n+1, n}$ denoting projection, is commutative.

(D) By the standard argument (for example, obstruction theory, as in [5]), define liftings $p_{n+1}^{X^{\prime}}: X^{\prime} \rightarrow X_{n+1}$ and $p_{n+1}^{Y^{\prime}}: Y^{\prime} \rightarrow Y_{n+1}$ subject to the conditions:

1. $\pi_{n+1, n}^{X} \cdot p_{n+1}^{X^{\prime}} \simeq p_{n}^{X^{\prime}} ; \pi_{n+1, n}^{Y} \cdot p_{n+1}^{Y^{\prime}} \simeq p_{n}^{Y^{\prime}}$.

2. $\left(p_{n+1}^{X^{\prime}} \mid F_{n}^{X}\right)^{*}$ and $\left(p_{n+1}^{Y^{\prime}} \mid F_{n}^{Y}\right)^{*}$ map the respective fundamental classes onto one another.

Now, consider the exact ladder( $\left.{ }^{4}\right)$

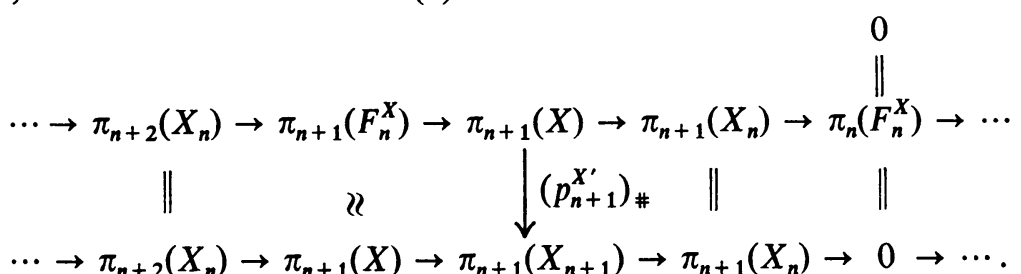

e see that $\left(p_{n+1}^{X^{\prime}}\right)_{\#}: \pi_{n+1}(X) \underset{\rightarrow}{\approx} \pi_{n+1}\left(X_{n+1}\right)$. More generally, we have $\left(p_{n+1}^{X^{\prime}}\right)_{\#}: \pi_{i}(X) \stackrel{\approx}{\rightarrow} \pi_{i}\left(X_{n+1}\right), i \leqq n+1 ;$ similarly, $\left(p_{n+1}^{Y^{\prime}}\right)_{\#}: \pi_{i}(Y) \stackrel{\approx}{\rightarrow} \pi_{i}\left(Y_{n+1}\right)$, $i \leqq n+1$.

Hence, the addition of the terms $X_{n+1}$ and $Y_{n+1}$ to the (finite) Postnikov systems $\left\{X, X_{n}, \cdots, X_{p}\right\}$ and $\left\{Y, Y_{n}, \cdots, Y_{p}\right\}$ gives us new Postnikov systems, each containing one more term.

(4) By the ACHP, we may assume $p_{n+1}^{X^{\prime}}$ and $p_{n+1}^{Y^{\prime}}$ to be fibre space maps. 
(E) It remains to arrange matters so that

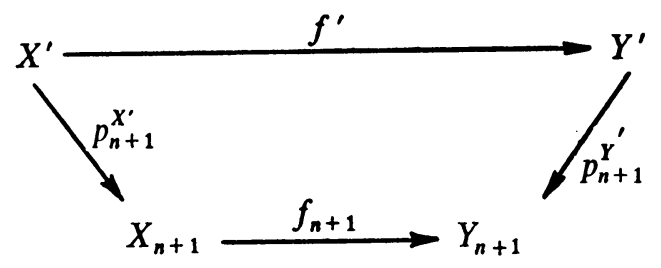

is homotopy commutative.

In the diagram

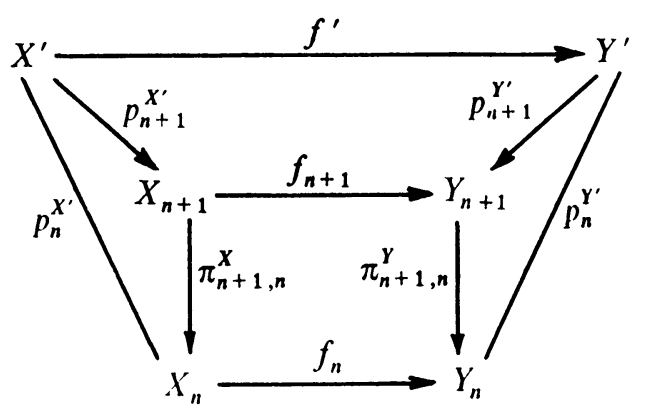

all diagrams, except possibly the top are commutative $\left({ }^{5}\right)$. Thus, we have

$$
\pi_{n+1, n}^{Y} \cdot f_{n+1} \cdot p_{n+1}^{X^{\prime}}=f_{n} \cdot p_{n}^{X^{\prime}}=p_{n}^{Y^{\prime}} \cdot f^{\prime}=\pi_{n+1, n}^{Y} \cdot p_{n+1}^{Y^{\prime}} \cdot f^{\prime} .
$$

It follows that there is $g: X^{\prime} \rightarrow K\left(\pi_{n+1}(Y), n+1\right)$ such that if $x \in X^{\prime}$,

$$
g(x) \cdot\left(f_{n+1} \cdot p_{n+1}^{X^{\prime}}(x)\right) \simeq p_{n+1}^{Y^{\prime}} \cdot f^{\prime}(x)\left({ }^{6}\right),
$$

and correspondingly a unique element $u \in H^{n+1}\left(X^{\prime}, \pi_{n+1}(Y)\right), \bar{u} \simeq g$. The map $p_{n+1}^{X^{\prime}}$ induces isomorphisms in homotopy in dimensions $\leqq n+1$, so that by Whitehead's theorem and the universal coefficient theorem (see [13] and [4]) it induces isomorphisms in cohomology with coefficients in $\pi_{n+1}(Y)$, in dimensions $\leqq n+1$. Now choose $v \in H^{n+1}\left(X_{n+1}, \pi_{n+1}(Y)\right)$ such that $\left(p_{n+1}^{X^{\prime}}\right)^{*} v=u$. By Lemma 1.3 , we have a homotopy commutative diagram

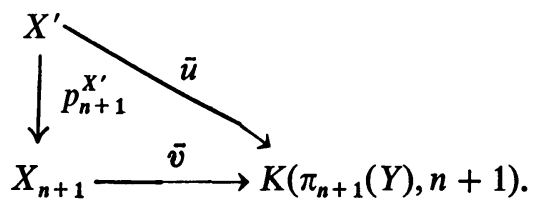

Define a new $\operatorname{map} f_{n+1}^{\prime}(x)=\bar{v}(x) \cdot f_{n+1}(x)$. In other words, $f_{n+1}^{\prime}$ is the composition

(5) We may assume that the triangular subdiagrams are (strictly) commutative, as the fibrations satisfy the ACHP.

(6) The multiplication in the left term is the action of the fibre. 
Proof. By converting $p_{n-1}$ into a fibre map, we get a homotopy commutative diagram

$$
X_{n+1} \stackrel{\Delta}{\longrightarrow} X_{n+1} \times X_{n+1} \stackrel{\bar{v} \times f_{n+1}}{\longrightarrow} K\left(\pi_{n+1}(Y), n+1\right) \times Y_{n+1} \stackrel{\mu}{\rightarrow} Y_{n+1}
$$

where $\Delta$ is the diagonal and $\mu$ is the action of the fibre. Then we see immediately that $f_{n+1}^{\prime}$ is also a fibre map in the sense that the rectangular diagram above is still commutative. Also,

$$
f_{n+1}^{\prime} \cdot p_{n+1}^{X^{\prime}}(x)=\bar{v}\left(p_{n+1}^{X^{\prime}}(x)\right) \cdot f_{n+1}\left(p_{n+1}^{X^{\prime}}(x)\right)
$$

while

$$
p_{n+1}^{Y^{\prime}} \cdot f^{\prime}(x) \simeq g(x) \cdot\left(f_{n+1} \cdot p_{n+1}^{X^{\prime}}(x)\right) .
$$

Since $g \simeq \bar{v} \cdot p_{n+1}^{X^{\prime}}$, we have $f_{n+1}^{X^{\prime}} \cdot p_{n+1}^{\prime} \simeq p_{n+1}^{Y^{\prime}} \cdot f^{\prime}$, as desired.

(F) To complete the proof, we rename $f_{n+1}^{\prime}$ as just $f_{n+1}$. Notice that $X^{\prime}$ was obtained from $X$ by replacing a map by a fibre map, that is points in $X^{\prime}$ consist of pairs $(x, p)$. Projection onto the first factor gives a canonical homotopy equivalence $h_{X}: X \rightarrow X^{\prime}$. Similarly, $h_{Y}: Y \rightarrow Y^{\prime}$. Of course,

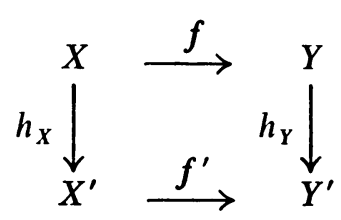

is homotopy commutative, so that we may replace $X^{\prime}$ by $X$, and $Y^{\prime}$ by $Y$, completing the induction step and the proof of the theorem.

REMARKS. In the proof of the above theorem, certain choices were made. These occurred in the construction of the Postnikov systems for $X$ and $Y$, at the point where we chose liftings of $p_{n}^{X^{\prime}}$ to $p_{n+1}^{X^{\prime}}$ (and similarly for $Y$ ). Apart from these choices, and up to suitable homotopies, the construction of the induced maps is uniquely defined. The next theorem is similar to Theorem 2.1 , except for the fact that the Postnikov systems for $X$ and $Y$ may be specified in advance. First we need a lemma.

Lemma 2.1. Let $X \in \mathscr{C}$ and $\left\{X_{i}, p_{i}, \pi_{i, j}\right\}$ be a Postnikov system for $X$. Consider the diagram

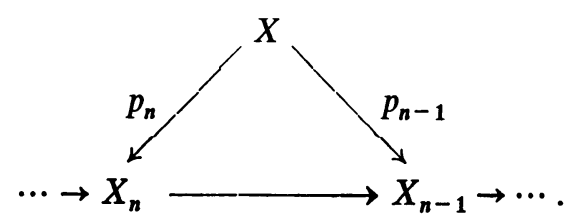

Suppose $p_{n-1}$ is converted into a fibre map, and write the characteristic class (image of the fundamental class under transgression) as $c^{n+1}$. Then $c^{n+1}=k^{n+1} \in H^{n+1}\left(X_{n-1}, \pi_{n}(X)\right)$. 


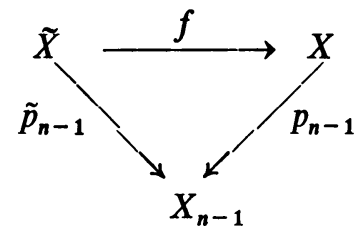

in which $\tilde{p}_{n-1}$ is a fibre map, and $f$ is a homotopy equivalence. By the ACHP, we may then assume

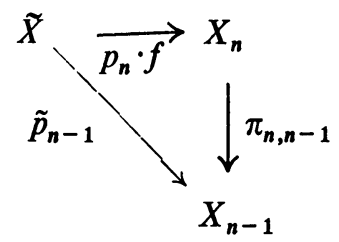

to be commutative, and we get a map of exact sequences:

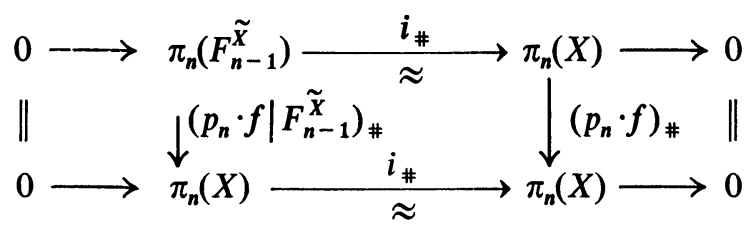

where $F_{n-1}^{\tilde{X}}$ denotes the fibre of $\tilde{p}_{n-1}$. It then is immediate that $p_{n} \cdot f \mid F_{n-1}^{\tilde{X}}$ induces an isomorphism in homotopy in dimension $n$. We identify these two groups; as the Hurewicz homomorphism is natural, it follows that $\left(p_{n} \cdot f \mid F_{n-1}\right)^{*}$ maps the fundamental class onto the fundamental class. Since the transgression is natural, it follows that $c^{n+1}=k^{n+1}$.

TheORem 2.2. Let $X, Y \in \mathscr{C}$, and let $f: X \rightarrow Y$ be a map. Suppose we are given Postnikov systems $\left\{X_{n}, p_{n}^{X}, \pi_{n, n-1}^{X}\right\}$ and $\left\{Y_{n}, p_{n}^{Y}, \pi_{n, n-1}^{Y}\right\}$ for $X$ and $Y$. Then there is a diagram

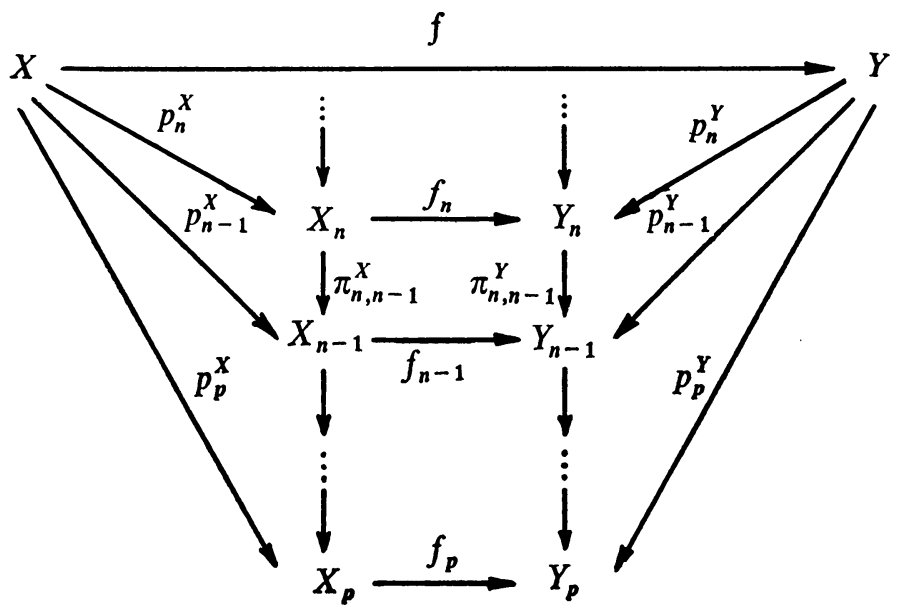


in which the rectangular diagrams are commutative, and all other diagrams are homotopy commutative. Furthermore, we have

$$
f_{c}^{*} k_{X}^{n}=f_{n-2}^{*} k_{Y}^{n}, \quad n \geqq p+2 .
$$

Proof. The proof will follow Theorem 2.1. First choose a map

$$
f_{p}: X_{p}=K\left(\pi_{p}(X), p\right) \rightarrow K\left(\pi_{p}(Y), p\right)=Y_{p}
$$

so that the following is homotopy commutative:

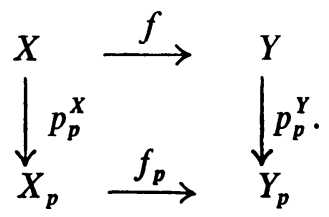

This may be done by choosing $f_{p}$ such that the two compositions induce the same map on homotopy in dimension $p$. By Proposition 2.3, the theorem is true for this system.

Now assume that it is true for the first $n-p$ terms of each system. The proof that the theorem is true for the first $n-p+1$ terms is also in steps.

(A) Same as Theorem 2.1.

(B) By Lemma 2.1 above, this is the same as in Theorem 2.1.

(C) Same as Theorem 2.1.

(D) Define the liftings to $X_{n+1}$ and $Y_{n+1}$ to be the liftings given by our original Postnikov systems.

(E \& F) Same as Theorem 2.1.

REMARK. Theorem 2.2 may be related to the question of when two maps are or are not homotopic. By way of an example, consider two spaces $X, Y \in \mathscr{C}$ which are finite CW-complexes. Fix Postnikov systems $\left\{X_{n}, p_{n}^{X}, \pi_{n, n-1}^{X}\right\}$ and $\left\{Y_{n}, p_{n}^{Y}, \pi_{n, n-1}^{Y}\right\}$. Then two maps $f, g: X \rightarrow Y$ are homotopic, if and only if the maps $f_{n}$ and $g_{n}$, considered as simplicial maps of simplicial complexes $X_{n}^{\prime}, Y_{n}^{\prime}$, which are equivalent to $X_{n}$ and $Y_{n}$, are homotopic when restricted to being maps of the $n$-skeleton of $X_{n}^{\prime}$ into the $n$-skeleton of $Y_{n}^{\prime}$.

3. Applications to $H$-spaces. In this section, I apply the results of $\S 2$ to $H$-spaces. We need the following lemma which follows immediately from the above material.

Lemma 3.1. Let $X$ have a Posinikov system $\left\{X_{n}, p_{n}, \pi_{n, n-1}\right\}$, with $k$-invariants $k_{X}^{m}$. Then $\left\{X_{n} \times X_{n}, p_{n} \times p_{n}, \pi_{n, n-1} \times \pi_{n, n-1}\right\}$ is a Postnikov system for $X \times X$, with invariants $i_{j c}^{*} p_{1}^{*} k_{X}^{m}+i_{2 c}^{*} p_{2}^{*} k_{X}^{m}$, where $p_{j}: X \times X \rightarrow X$ are the projections and where $i_{1 c}^{*}$ and $i_{2 c}^{*}$ are induced by the inclusions $i_{j}: X \rightarrow X \times X, j=1,2$.

In the definition of an $H$-space, one ordinarily imposes some condition on the existence of a unit or homotopy unit. These conditions are equivalent for 
complexes. For what will follow, we will use a slightly different definition given below. Such spaces will be called almost $H$-spaces ( $A H$-spaces). Proposition 3.1 shows that they are the same as $H$-spaces in the cases which are of interest to us. These spaces were essentially considered in [1].

Definition 3.1. A space $X$ is called an $A H$-space if there is a map $\mu: X \times X \rightarrow X$ so that if we denote by $i_{j}: X \rightarrow X \times X, j=1,2$, the inclusions, then $\mu \cdot i_{1}$ and $\mu \cdot i_{2}$ are homotopy equivalences. If $X$ and $Y$ are $A H$-spaces, then a map $f: X \rightarrow Y$ is called an $A H$-map, if the diagram

is commutative.

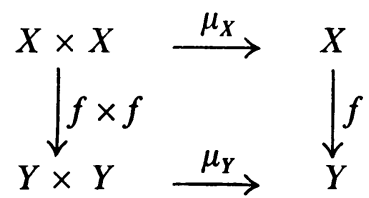

TheOREM 3.1. Let $X \in \mathscr{C}$ be an AH-space, $\left\{X_{n}, p_{n}, \pi_{n, n-1}\right\}$ be a Postnikov system for $X$. Then each space $X_{n}$ may be given the structure of an AH-space in such a way that the maps $\pi_{i, j}, i>j$, are then all AH-maps.

Proof. By Theorem 2.2 and Lemma 3.1, we have a diagram

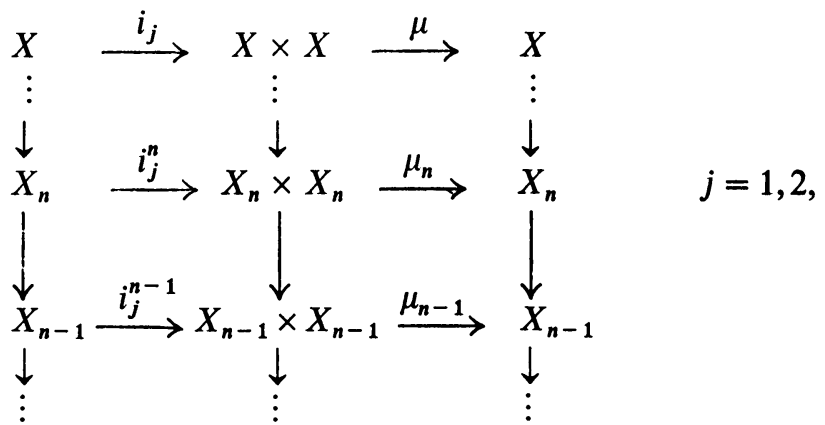

where $i_{j}^{n}$ designates the inclusion as the $j$ th factor $(j=1,2)$ in the product, and where all subdiagrams involving the top row are homotopy commutative, while the other subdiagrams are strictly commutative. I have omitted the maps $p_{n}$, etc. from the picture. Now the map $\mu \cdot i_{j}$ induces isomorphisms in homotopy in all dimensions. From the diagram,

or thus

$$
\mu_{n} \cdot i_{j}^{n} \cdot p_{n} \simeq p_{n} \cdot \mu \cdot i_{j}
$$

$$
\left(\mu_{n} \cdot i_{j}^{n}\right)_{\#} \cdot\left(p_{n}\right)_{\#}=\left(p_{n}\right)_{\#} \cdot\left(\mu \cdot i_{j}\right)_{\#} \cdot
$$

In dimensions $\leqq n,\left(p_{n}\right)_{\neq}$is an isomorphism. Hence, $\left(\mu_{n} \cdot i_{j}^{n}\right)_{\#}$ is an isomorphism in this range. But if $j>n, \pi_{j}\left(X_{n}\right)=0$. Hence, $\mu_{n} \cdot i_{j}^{n}$ induces isomorphisms of all homotopy groups. As $X_{n}$ has the homotopy type of a complex, we conclude that $\mu_{n} \cdot i_{j}^{n}$ is a homotopy equivalence. The diagram shows that $\pi_{i, j}$ is an $A H$-map. 
The principal interest in $A H$-spaces is perhaps the following proposition which is due to A. Dold.

Proposition 3.1. Let $X$ be a 0-connected AH-space. Then $X$ is an H-space for some possibly different multiplication.

Proof. Let $e$ be the base point of $X$, and denote

$$
L=\mu \cdot i_{2} \quad R=\mu \cdot i_{1} .
$$

By hypothesis, $L$ and $R$ are homotopy equivalences. Let a left homotopy inverse for $L$ be written $L^{-}$and a right homotopy inverse for $R$ be written $R^{-}$.

Define

Then

$$
v: X \times X \rightarrow X \text { by } v(y, z)=L^{-}\left[\mu\left(R^{-} L(y), z\right)\right]
$$

$$
\begin{aligned}
v(e, z) & =L^{-}\left[\mu\left(R^{-} L(e), z\right)\right]=L^{-}\left[\mu\left(R^{-1} \mu(e, e), z\right)\right]=L^{-}\left[\mu\left(R^{-} R(e), z\right)\right] \\
& \simeq L^{-}[\mu(e, z)]=L^{-} L(z) \simeq z
\end{aligned}
$$

while

$$
v(y, e)=L^{-}\left[\mu\left(R^{-} L(y), e\right)\right]=L^{-}\left[R R^{-} L(y)\right] \simeq L^{-} L(y) \simeq y\left({ }^{7}\right) .
$$

Corollary 3.1. Let $X \in \mathscr{C}$ have a Postnikov decomposition $\left\{X_{n}, P_{n}, \pi_{n n-1}\right\}$. If $X$ is an $H$-space, then each $X_{n}$ is an H-space.

Corollary 3.2. Let $X \in \mathscr{C}$ have a Posinikov system $\left\{X_{n}, p_{n}, \pi_{n, n-1}\right\}$. Let $k$ be a perfect field. If $X$ is an $H$-space, then for each $n, H^{*}\left(X_{n} ; k\right)$ is a tensor product of (possibly truncated) polynomial algebras on one generator.

Proof. Apply Borel's theorem [1, Theorem 6.1] to Corollary 3.1.

Our final application will be to characterize $H$-spaces with finitely-many nonvanishing homotopy groups (compare [3]). Let $X$ be an $A H$-space with multiplication map $\mu: X \times X \rightarrow X$. Consider the natural splitting

$$
H^{n}(X \times X ; G) \approx H^{n}(X \vee X ; G) \oplus H^{n}(X \times X, X \vee X ; G)
$$

for any Abelian group $G$.

Denote by $\sigma: H^{n}(X \times X ; G) \rightarrow H^{n}(X \times X, X \vee X ; G)$ the projection onto the second summand. We make the following definition:

Definition 3.2. A cohomology class $u \in H^{n}(X ; G)$ is called $G$-primitive, if $\sigma \cdot \mu^{*}(u)=0$.

THEOREM 3.2. Let $X$ be a space with finitely-many nonvanishing homotopy groups, $\pi_{n_{i}}, \cdots, \pi_{n_{k}}$, where $\pi_{n_{i}}=\pi_{n_{i}}(X)$ and $n_{1}<\cdots<n_{k}$. Then $X$ is an $H$-space, if and only if it admits a Postnikov decomposition for which each $k$-invariant, $k^{n_{i}+1}$, is $\pi_{n_{i}}$-primitive, with respect to the map $\mu_{n_{i}}$ (as in the previous theorem)( $\left.{ }^{8}\right)$.

(7) For convenience, we write the maps acting on elements of $X \times X$.

(8) This is, of course, an inductive condition for such a space to be an $H$-space. 
Proof. Suppose, first of all, that each $k$-invariant is primitive in the above sense. We shall show that if the term $X_{n_{i}-1}$ is an $H$-space, and the element $k^{n_{i}+1}$ is $\pi_{n_{i}}$-primitive, then $X_{n_{i}}$ is an $H$-space. Since $X_{n_{i}}=K\left(\pi_{n_{i}}, n_{1}\right)$ is clearly an $H$-space the first part of the theorem will follow by induction.

Consider the diagram

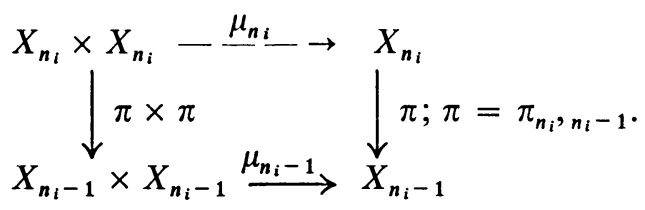

We desire to fill in the top row. Without destroying the homotopy commutativity of the final diagram, we may suppose that $X_{n_{i}} \times X_{n_{i}}$ is a CW-complex, with the $X_{n_{i}} \vee X_{n_{i}}$ a sub complex. Furthermore, we may suppose $\pi: X_{n_{i}} \rightarrow X_{n_{i}-1}$ to be a locally trivial fibre map.

It is easy to see that we may lift the map $\mu_{n_{i}-1} \cdot(\pi \times \pi)$ over the $n_{i}$-skeleton to $\bar{\mu}_{n_{i}}:\left(X_{n_{i}} \times X_{n_{i}}\right)^{n_{i}} \rightarrow X_{n_{i}}$. The obstruction to extending $\bar{\mu}_{n_{i}}$ to the $\left(n_{i}+1\right)$-skeleton is $(\pi \times \pi)^{*} \cdot \mu_{n_{i}-1}^{*}\left(k^{n_{i}+1}\right)$. Since $\mu_{n_{i}-1}$ is an $H$-space map, and $k^{n_{i}+1}$ is $\pi_{n_{i}}$-primitive, $\mu_{n_{i}-1}^{*}\left(k^{n_{i}+1}\right)$ lies in $H^{n_{i}+1}\left(X_{n_{i}-1} \vee X_{n_{i}-1} ; \pi_{n_{i}}\right)$ and in fact is $k^{n_{i}+1} \vee k^{n_{i}+1}$ in $H^{n_{i}+1}\left(X_{n_{i}-1}, \pi_{n_{i}}\right) \oplus H^{n_{i-1}}\left(X_{n_{i}-1} ; \pi_{n_{i}}\right)$ identified with $H^{n_{i+1}}\left(X_{n_{i}-1} \vee X_{n_{i}-1}, \pi_{n_{i}}\right)$ As $\pi^{*}\left(k^{n_{i}-1}\right)=0$, we see that $(\pi \times \pi)^{*} \mu_{n_{i}-1}^{*}\left(k^{n_{i}+1}\right)=0$. Hence, $\bar{\mu}_{n_{i}}$ may be extended over the $\left(n_{i}+1\right)$-skeleton, and hence, to all of $X_{n_{i}}$. Denote this map by $\mu_{n_{i}}$. Now, $\mu_{n_{i}}$ need not be an $H$-space map. However, we may require that $\mu_{n_{i}}$ restricted to the subcomplex $X_{n_{i}} \vee X_{n_{i}}$ be homotopic to the folding map $X_{n_{i}} \oplus X_{n_{i}} \rightarrow X_{n_{i}}\left({ }^{9}\right)$, and then the compositions

$$
X_{n_{i}} \stackrel{i_{j}^{n_{i}}}{\longrightarrow} X_{n_{i}} \times X_{n_{i}} \stackrel{\mu_{n_{i}}}{\longrightarrow} X_{n_{i}}
$$

will be homotopic to the identity.

To prove the converse, let $\left\{X_{n}, p_{n}, \pi_{n, n-1}\right\}$ be the Postnikov system and consider the corresponding $A H$-space structure on each $X_{n}$ given by Theorem 3.1.

We have immediately

and applying Lemma 3.1,

$$
\mu_{n_{i}}^{*} k_{X}^{n_{i}+2}=\mu_{c}^{*} k_{X \times X}^{n_{i}+2}
$$

$$
\mu_{n_{i}}^{*} k_{X}^{n_{i}+2}=\mu_{c}^{*} \cdot i_{1 c}^{*} \cdot p_{1}^{*} k_{X}^{n_{i}+2}+\mu_{c}^{*} \cdot i_{2 c}^{*} \cdot p_{2}^{*} k_{X}^{n_{i}+2} .
$$

As $\mu \cdot i_{1}$ and $\mu \cdot i_{2}$ are homotopy equivalences, we see that the last expression belongs to

$$
H^{n_{i}+2}\left(X_{n_{i}} ; \quad \pi_{n_{i}+1}\right) \oplus H^{n_{i}+2}\left(X_{n_{i}} ; \quad \pi_{n_{i}+1}\right)=H^{n_{i}+2}\left(X_{n_{i}} \vee X_{n_{i}} ; \pi_{n_{i}+1}\right)
$$

and is thus annihilated by $\sigma$.

(9) As $H^{n_{i}+1}\left(X_{n_{i}-1} \times X_{n_{i}-1}, X_{n_{i}-1} \vee X_{n_{i}-1} ; \pi_{n_{i}}\right) \rightarrow H^{n_{i}+1}\left(X_{n_{i}-1} \times X_{n_{i}-1} ; \pi_{n_{i}}\right)$ is a monomorphism, the (relative) obstruction must also vanish. 
Remark. The first part of the theorem (sufficient condition for such a space to be an $H$-space) is well known (compare [16]). The second part is true without the assumption of finitely-many nonvanishing homotopy groups. If one could express $X$ as a limit of the $X_{n}$, then one could prove the first part without the assumption of finitely-many nonvanishing homotopy groups $\left({ }^{10}\right)$.

\section{BIBLIOGRAPHY}

1. A. Borel, Sur la cohomologie des espaces fibrés principeaux et des espaces homogènes de groupes de Lie compacts, Ann. of. Math. (2) 57 (1953), 115-207.

2. H. Cartan, Séminaire 7, Secréteriat mathématique, Paris, 1955/56.

3. A. Copeland, Jr., On H-spaces with two non-trivial homotopy groups, Proc. Amer. Math. Soc. 8 (1957), 184-191.

4. S. Eilenberg and N. Steenrod, Foundations of algebraic topology, Princeton Univ. Press, Princeton, N. J., 1952.

5. R. Hermann, Secondary obstructions for fibre spaces, Bull. Amer. Math. Soc. 65 (1959), 5-8.

6. P. Hilton, and S. Wylie, Homology theory, Cambridge Univ. Press, Cambridge, 1961.

7. J. Milnor, The geometric realization of a semi-simplicial complex, Ann. of Math. (2) 65 (1957), 357-362.

8. - Construction of universal bundles. II, Ann. of Math. (2) 63 (1956), 430-436.

9. - On spaces having the homotopy type of a CW-complex, Trans. Amer. Math. Soc. 90 (1959), 272-280.

10. J. Moore, Semi-simplicial complexes and Postnikov systems, Symposium Internacional de Topologia Algebraica, Mexico, 1958.

11. F. Peterson and E. Thomas, A note on non-stable cohomology operations, Bol. Soc. Mat. Mexicana (1958).

12. M. Postnikov. Investigations in homotopy theory of continuous mappings, Trudy. Mat. Inst. Steklov. no. 46, Izdat. Akad. Nauk. SSSR, Moscow, 1955.

13. J.-P. Serre, Groupes d'homotopie et classes de groupes abéliens, Ann. of Math. (2) 58 (1953), 258-294.

14. —_ Homologie singulière des espaces fibrés. Applications, Ann. of Math. (2) 54 (1951), 425-505.

15. - Cohomologie modulo 2 des complexes d'Eilenberg-MacLane, Comment. Math. Helv. 27 (1953), 198-232.

16. H. Suzuki, Multiplications in Postnikov systems and their applications, Tôhoku Math. J. 12 (1960).

17. R. Thom, Quelques propriétés globales des variétés différentiables, Comment. Math. Helv. 28 (1954), 17-86.

18. J.H.C. Whitehead, Combinatorial homotopy. I, Bull. Amer. Math. Soc. 55 (1949) 213-245.

\section{COLUmbia University,} New YORK, NeW YoRK

(10) After this paper was written, I learned from M. G. Barratt that one can show, by a semisimplicial argument, that the theorem is true in general. 